\title{
On the Mechanism of Atmospheric Vortex Formation and How to Weaken a Tornado
}

\author{
Andrei Nechayev and Alexander Solovyev
}

\section{ABSTRACT}

\begin{abstract}
A hydrodynamic mechanism of tornado formation is proposed: it is based on the air density decrease in ascending jets. The empirical verification of this mechanism is considered. The ways are discussed to weaken the tornado by reducing the inflow streams humidity or by introducing the discontinuity of air jets connecting the lower and upper layers of the atmosphere.
\end{abstract}

Keywords: atmospheric vortex, hurricane, waterspout, tornado
Published Online: December 25, 2019

DOI : $10.24018 / 2019.1 .1 .1$

A. M. Nechayev*

Lomonosov's Moscow State University, Geographic Department, Russia.

(e-mail: and.nechayev@gmail.com)

A. A. Solovyev

Lomonosov's Moscow State University,

Geographic Department, Russia.

(e-mail: geosolmgu@mail.ru)

*Corresponding Author
The atmospheric vortex, as a natural phenomenon, has long been known. Its most formidable varieties - such as tornadoes and hurricanes - are too familiar to many inhabitants of our planet, as they bring them troubles and troubles. Modern methods of photo and video fixation contain colossal information about atmospheric vortices from the tiny and harmless dust "devils" to deadly tornadoes that destroy everything in their path, and hurricanes that flood vast territories with rain. A lot of scientific work in the field of meteorology and atmospheric physics is devoted to vortices, the empirical laws of their behavior are quite well established and described [1]. As for theoretical works, the most cited ones [2-4] liken the atmospheric vortex to the Carnot heat engine, deriving the condition for its formation from thermodynamic relations and the integral laws of conservation of energy, mass, angular momentum. Meanwhile, the atmospheric vortex is a fundamentally open hydrodynamic system, to which conservation laws are not always applicable. In any case, the physically clear and simple mechanism of atmospheric vortex formation, unfortunately, is absent.

In [5] it was demonstrated that many of the so-called hydrodynamic "paradoxes" can have a simple explanation in the framework of classical hydrodynamics, if its laws are used correctly. Here we will try to use a similar approach to explain the observed atmospheric phenomenon, which is still mysterious. The desire to find and understand a certain "universal" mechanism for the formation of an atmospheric vortex grows stronger when it becomes obvious that almost all natural vortices, regardless of their scale, have many common properties. The main ones are as follows:

1. The lower part of the axis of the atmospheric vortex, around which the air rotates, is always at a minimum of atmospheric pressure;

2. Air in a vortex moves in an ascending spiral, from bottom to top;
3. The intensification of the vortex is accompanied by an acceleration of air rotation and a drop in pressure in the center;

4. The origin of a natural vortex can occur in a relatively calm atmosphere.

5. An atmospheric vortex is formed in the Earth's atmosphere, which is barotropic: the air density in it depends on pressure and usually decreases with height.

In this paper, we will develop the new hydrodynamic mechanism proposed in [6] in order to analyze certain hydrodynamic factors that can lead to the formation of stable zones of reduced pressure in the earth's atmosphere, inside and around which an upward air movement occurs. The essence of this mechanism is as follows.

Imagine a pipe with expansion and a fluid flowing through the pipe, which, in the place of expansion, changes its density from $\rho_{1}$ to $\rho_{2}: \rho_{2}<\rho_{1}$. The expansion is chosen in a special way so as to ensure the constancy of the speed $v$ based on the condition of mass conservation $\rho v S=$ const where $S$ is the pipe section. Thus, the cross section of the pipe "tracks" the density of the fluid, keeping the flow rate constant, since $\rho S=$ const.

Let us write the Bernoulli equation for one-dimensional fluid flow along a streamline with a coordinate $s$. Let us proceed from the generalized form of Newton's second law for a fluid with variable density (in the absence of mass forces):

$$
\frac{D(\rho v)}{D t}=-\frac{\partial p}{\partial s}-F_{f r}(v),
$$

where $p$ is the pressure inside the fluid, $v$ is the linear velocity $(v=\partial s / \partial t), F_{f r}$ is the friction force acting on the fluid particle. The pressure at the ends of the pipe $p_{0}$ and $p_{2}$ is kept constant. Going to the stationary state $(\partial(\rho v) / \partial t)$, we can write: 


$$
\frac{\partial(\rho v)}{\partial s} v=-\frac{\partial p}{\partial s}-F_{f r}(v)
$$

In the first section $L_{1}$ where $\rho=\rho_{1}$, the pressure drops from $p_{0}$ to $p_{1}$, and the speed increases from 0 to $v$, integration (2) gives the standard Bernoulli equation with friction:

$$
\left(p_{0}-p_{1}\right)=\frac{\rho_{1} v^{2}}{2}+\bar{F}_{f r 1} L_{1}
$$

Integrating (2) over a segment of length $L_{2}$ where the density decreases and the speed remains constant, we have:

$$
\left(p_{1}-p_{2}\right)=\left(\rho_{2}-\rho_{1}\right) v^{2}+\bar{F}_{f r 2}(v) L_{2}
$$

Thus, for a fluid flow with a variable density, one can expect the appearance of a zone of reduced pressure in those places where the density begins to decrease. This pressure drop is greater, the greater the difference in densities and the velocity of the fluid. Friction weakens this effect. From (4) it follows that there will be no pressure drop $\left(p_{1} \geq p_{2}\right)$ if the friction in the second section is sufficiently large. If we neglect the friction forces (which is permissible for air), then it can be shown that the described system has instability with positive feedback between the flow velocity and the pressure drop. Indeed, summing (3) and (4) and neglecting friction, we obtain equation (5) for the flow velocity at the point where the density begins to decrease:

$$
p_{0}-p_{2}=\left(\rho_{2}-\frac{\rho_{1}}{2}\right) v^{2}
$$

From this equation it follows that velocity tends to infinity when the density decreases by half. However, this "catastrophe" will not happen if we take into account friction factors in (3) and (4). The velocity will not increase indefinitely, and the pressure $p_{1}$ will not drop to zero. The frictional forces, which we neglected for clarity, prevent this. Indeed, the friction forces $F_{f r}$ increase with velocity increasing and, as follows from (4), friction in the second section will reduce the pressure drop, which should stabilize the situation. The knowledge of the dependences $F_{f r}(v)$ allows one to solve equations (3) and (4) and obtain an unambiguous value of the flow velocity $v$ and pressure $p_{1}$ at given values $\rho_{1}, \rho_{2}$. The described instability mechanism is fundamental, since a decrease in the momentum $\rho v$ due to a decrease in density must be compensated by the pressure forces in accordance with the laws of hydrodynamics and leads to a pressure drop.

Before applying the results obtained above to a free atmosphere, we briefly discuss the possible mechanisms of ascending air movements. It is well known that vertical air currents, both ascending and descending, are one of the main driving forces for the formation of weather and climate on Earth. An intense and local rise in humid air accompanies the formation of thunderstorm fronts, squall lines and tornado clouds. Significantly weaker, but prolonged and large-scale ascending air movements in clusters above the warm ocean end under certain conditions with the formation of tropical storms and hurricanes.

Classical free convection in the atmosphere leads to noticeable rates of rise only in the case of closed volumes of light gas with a density many times lower than the density of the surrounding air. This happens, for example, in the case of stratospheric balloon filled with helium. Actually convective rise of hot air has a relatively low speed, as it is easy to notice when observing the flames of a fire (temperature above 1000 C) or ordinary water vapor (temperature $100 \mathrm{C}$ ) rising above a boiling pan. Obviously, a mechanism other than thermal convection acts in the free atmosphere, which creates in the lower and middle layers of the troposphere quite intense and long-lived zones of air rise with speeds exceeding units and even tens of meters per second. These zones are well known to passengers of airliners falling into air holes, parachutists and gliders who are near the base of a thundercloud, tourists and climbers on the slopes of mountain peaks. In all these cases, there are practically no noticeable temperature differences. Therefore, the mechanism of lifting air should be due to hydrodynamic reasons.

There is an obvious, but not always recognized feature of the behavior of the volumes of air inside the atmosphere. The weight of these volumes is balanced by buoyancy, and when the temperatures are equal, they are in indifferent equilibrium. Prandtl in his famous work [7] wrote about the excess pressure arising in a liquid as a result of its motion. This pressure "... is distributed in the liquid as if it was weightless and had only an inert mass ... Each particle of a heavy liquid hangs in a stream under the action of the supporting forces of the surrounding particles." Any, the slightest, force applied to the volume in the vertical direction (no matter up or down) sets it in motion just as if this force was applied horizontally. The gravitational force is balanced by a vertical gradient of hydrostatic pressure, and a balloon filled with room air moves equally easily both to the side and to the ceiling.

Therefore, considering the movement of air directed vertically upwards, we can "forget" about the weight of the air, since it is compensated by the Archimedean force, and assume that the initial pressure at all altitudes is "conditionally" the same and equal to some reference pressure $\bar{p}$. And the beginning of the movement is caused by primary overpressure. It is important for us that there is a certain force that can make air move upward, to an area where the air density is much less. The source of such a force can be the inhibition of air flow due to various reasons. When the flow velocity decreases to zero in the zone of collision with a fixed surface at the so-called critical point [5], the pressure increases by an amount $\rho v^{2} / 2$. This overpressure either stops the flow or causes it to change direction in accordance with the surrounding conditions. In a free atmosphere, a similar situation can arise, for example, if oncoming flows collide. In this case, mutual inhibition of both flows is inevitable with the formation of an overpressure zone, which forces air to move in the vertical direction. Collision of oncoming flows takes place in the case of the movement of air masses inside the zone of low pressure, which can form near the surface of the Earth due to hydrostatic reasons. So, for example, the characteristic pressure drop in the center of the tornado causes powerful centripetal flows, which mutually slow down and easily go up, because for them this is the only free direction, and the weight of the air, as noted above, does not interfere to the rise. This excess pressure causes air to move to the upper atmosphere, where the density of air is substantially lower. Thus, a stream of air with a variable density can form, which 
will lead to a drop in pressure in the lower part of the jet in accordance with the mechanism described above. The condensation of water vapor accompanied by the release of latent heat should lead to further reduce of the density of air. Indeed, during the condensation of water vapor, heat is released, which is determined by the latent heat of condensation and air humidity. If this heat exceeds adiabatic air cooling (10 degrees per $1 \mathrm{~km}$ of lift), then a particle of air acquires heat $\Delta T$, which, in accordance with the equation of gas state, reduces its density by the value $p \Delta T / R T^{2}$, where $p$ is the pressure at a given height, $T$ is the air temperature, $R$ is the universal gas constant. If the density of air in the jet decreases, the volume and cross-section of the air jet proportionally increases (the jet expands), which can lead to a constant air velocity in the jet, similar to the above thought experiment. In support of this assumption, we can mention the well-known fact of maintaining to very high altitudes almost constant values of the tangential velocity of air jets rising in the wall of the eye of a hurricane [8].

Thus, from a hydrodynamic point of view, the formation of an atmospheric vortex can occur when conditions are created for the pressure rise of the air mass (jet) into the upper atmosphere, where the air density decreases in accordance with the equation of state. Water vapor condensation leads to an additional decrease in density. The vortex intensification can be expected when the air density of the jet, when it rises, decreases at least by half.

Now we give an interpretation of some empirical data on real atmospheric vortices. To do this, we use the formula (4), simplified by the absence of friction, which connects the pressure drop $\Delta p$ in a vortex with the drop in air density in the jet and its speed $v$ :

$$
\Delta p=-v^{2} \Delta \rho
$$

where $\Delta \rho$ is the difference between the density of air at the entrance to the vortex and where the jet ceases to exist.

The easiest way to explain the formation of tornadoes during major forest fires or volcanic eruptions. Indeed, a column of hot air with a temperature above $600 \mathrm{~K}$ (at such a temperature the air density decreases by 2 times) satisfies instability criterion $\left(\rho_{2}<\rho_{1} / 2\right)$ and creates a primary hydrostatic pressure drop (albeit small), which "draws" into the column the cold and "dense" ambient air, actually simulating the conditions of our thought experiment.

The origin of the dust "devil" can also be due to air heating. In desert areas where "devils" usually form, local heating of surface air to temperatures of 50-60 C is common. If air with such a temperature forms a layer hanging or floating above the ground, then the air density in it will be less than the density of the surrounding air. If a wind stream with a speed of 10-20 m/s falls into such a "hot" layer, then, according to (6), a pressure drop of $0.2-0.8 \mathrm{mB}$, which is typical for small "devils", will be on the "cold" end of the stream.

In powerful hurricanes, streams of humid air rise in a spiral to heights of $10-12 \mathrm{~km}$, maintaining a very high speed, up to $50-60 \mathrm{~m} / \mathrm{s}$. The difference in $\Delta \rho$ can reach $0.8-1.0$ $\mathrm{kg} / \mathrm{m}^{3}$. For example, in Hurricane Inez (1966) the recorded maximum wind speed $v_{m}$ was $80 \mathrm{~m} / \mathrm{s}$ [8]. Formula (6) gives us an estimate of the pressure drop in the eyewall (and not in the center of the hurricane's eye) where the air flow begins to rise: $51-64 \mathrm{mB}$. To estimate the pressure in the center of the hurricane's eye, one should add to this value a value $\rho v_{m}^{2} / 2$ that reduces the pressure in the center of the rotating air "disk": the "solid-state" rotation of the hurricane's eye with angular velocity $v_{m} / R_{m}$ (where $R_{m}$ is the Radius of Maximum Wind) is well established. Consequently, the average total pressure drop in the center of Hurricane Inez will be about $92 \mathrm{mB}$ (the air density in the RMB area is assumed to be $1.1 \mathrm{~kg} / \mathrm{m}^{3}$ ), which corresponds to the actually recorded minimum pressure of $927 \mathrm{mB}(927+92=1019$ $\mathrm{mB})[8]$.

From the very beginning of focused research on tropical cyclones, scientists were faced with the task of obtaining a ratio between the maximum wind speeds and the minimum value of atmospheric pressure. This relationship has been established, mainly, empirically in the form of a power dependence $v_{m}=C(\Delta p)^{n}$, where $n<1[9,10]$. The most successful formula is currently attributed to Willoughby [11]:

$$
v_{m}=\sqrt{\frac{2}{3} \frac{1}{\rho}\left(p_{\infty}-p_{c}\right)}
$$

where $p_{\infty}$ and $p_{c}$ are pressure outside the hurricane and in its center, respectively.

Our theoretical assumptions give the following formula:

$$
\left(\rho_{1}-\rho_{2}\right) v_{m}^{2}+\rho_{1} v_{m}^{2} / 2=p_{\infty}-p_{c}
$$

It almost coincides with (7), if we assume $\rho_{2}<<\rho_{1}$ that is typical of hurricanes, in which air jets rise to a height of at least $10 \mathrm{~km}$.

Thus, for the formation of an atmospheric vortex, a primary jet is necessary, having a drop $\Delta \rho$ in air density sufficient to initiate a positive feedback in accordance with equations (4) and (5). Inside this jet, a section of pressure reduced to the adjacent horizontal layers is realized which causes circulation around the stream: the rotation of ambient air. It is known that the rotation of air in the mesocyclone is always accompanied by its rise in the center of the vortex. The "embryos" of tropical cyclones, tropical clusters, must have the circulation, and the primary updraft flow in these clusters reaches the high layers of the atmosphere with low air density. The condition $\left(\rho_{2}<\rho_{1} / 2\right)$ of the intensification of the atmospheric vortex will be satisfied if the primary jet reaches a 6-kilometer height. Another necessary condition for the formation of a cyclone from a tropical cluster is the absence of vertical wind shear. Otherwise, the continuity of the primary jet will be broken, and the above feedback mechanism will not work.

Hurricanes are formed over the flat surface of the ocean as well as waterspouts. Tornadoes "love" flat spaces of land. Everywhere there is humid air. The presence of a cold front facilitates the rise of warm air in the upper atmosphere.

Hurricanes weaken when they land and go deeper into northern latitudes. This is because the incoming air has less moisture than warm air above the ocean. Less humid, or even dry, the air can't rise to significant heights and form a jet with a large density difference $\Delta \rho$.

In a free atmosphere, air rotating around the primary jet plays the role of pipe walls: the centrifugal force of rotation compensates the pressure drop between the axis of the jet and the periphery. These spiraling upward, secondary jets take 
over the function of the primary jet, connecting the layers of the atmosphere with different air density. Along these jets, a reduced pressure is transmitted to the lower layers of the atmosphere.

The tangential velocity of the secondary jets due to viscosity initiates the "solid-state" rotation of the central core of the vortex and reduces the pressure on its axis. As a result, the primary jet actually ceases to exist, forming the eye of a hurricane or the core of a tornado, in which there is a downward movement of air.

Tornado is one of the most impressive varieties of atmospheric vortex. It, unlike a hurricane, is visible to the naked eye. Pressure dips and wind speeds in powerful tornadoes tend to exceed those for hurricanes. And here, in connection with the proposed mechanism, new questions arise that are not yet answered. First, the air comes to the forefront, where the law of an ideal gas must be in effect, connecting the gas pressure and its density. If the air stream connected areas with different air densities and a pressure drop occurred in it, then the air density should decrease in this place. Accordingly, an additional mechanism of positive feedback appears, which can enhance the process of pressure drop in the air. Perhaps this can explain the well-known lowering of the tornado funnel to the ground and the spread of rotation to the surface area. Secondly, the initiation of a tornado and similar water tornadoes begins with the rise of air inside the central core [12]. This fact is in good agreement with our theory, because the jet in the central core effectively connects atmospheric layers with different air densities. A single hydrodynamic stream allows us to explain the often observed bizarre shape of a tornado, its curved thin "trunk" touching the ground and rotating at high speed. Our mechanism does not need verticality. It is not hydrostatic. A jet, that is, a streamline, can have any shape: it is important that it does not have gaps and that it connects a region with a high air density (Earth's surface) to a region where the air density is low (upper atmosphere).

The rise of air in the central core also occurs during the formation of a hurricane: they arise in clusters after a long rise in moist air in the center of very weak circulation (with wind speeds of the order of 1-2 m/ s), which then intensifies. However, the rotation in a mature hurricane is integral: the individual cumulus clouds-towers that form the hurricane themselves do not rotate, they move collectively around the eye of the hurricane, where there is practically no air movement. Perhaps in a hurricane a single stream of air exists only at the beginning of formation, at the stage of a tropical storm. However, in the tornado there is a "core", the space inside the funnel, which replaces the primary single stream. By the way, hurricanes, as you know, are able to give birth to tornadoes upon entering land [13]. Perhaps their structure (the rise of air to the tropopause regions, high wind speeds) facilitates the formation of pressure zones, where vertical jets of air are formed that can rise up and reach the upper words of the atmosphere.

The primary jet, and then the secondary helical jets are the "arteries" of the tornado, feeding it with moist air. It is known that the cooled and dry air, penetrating the waterspout, leads to its destruction [12]. Obviously, the jets, devoid of moisture, can't reach high altitudes with low air density. In this case, perhaps, the remedy against a tornado is any hygroscopic powder, for example, sodium iodide, injected into the jet at the entrance to the vortex. Since the jets in the tornado do not mix, the effectiveness of the hygroscopic powder will be limited by the jet section into which it fell. Therefore, the process of injecting the powder inside the tornado should not be single. It is necessary to process all tornado streams (there may be more than a dozen of them) and as many areas as possible. Since tornado jets will not be able to rise to great heights, the hydrodynamic connection between the lower and upper layers of the atmosphere will be broken. The mechanism of pressure drop in the jet due to the difference of air density will stop working: the pressure in the center of the tornado will start to grow, the wind speed will decrease.

There may be a lot of technical methods for delivering powder inside a tornado. For example, it can be projectile, rocket or firecracker, equipped with a hygroscopic powder.

The continuity of the ascending streams of a tornado can be broken by firing blank cartridges. Excessive pressure around the point of rupture should break up the streamline of the jet, break its hydrodynamic connection with the upper layers of the atmosphere. It is only important that the area of the explosion overlaps the cross section of the jet. Of course, one shot will not be enough. A tornado, like a multi-headed "hydra", will not die if it has only one "head-jet" cut off. All streams should be broken and preferably in several places.

Waterspouts can serve as a test object for the methods described above. "Shelling" of such a vortex can be effected both from an airplane and from a sea vessel.

\section{REFERENCES}

[1] Superstorm Encyclopedia: Tornadoes, Severe Thunderstorms, Hurricanes, Tropical Storms, Typhoons, Cyclones ... Progressive Management, 2011.

[2] K.A.Emanuel, "An air-sea interaction theory for tropical cyclones. Part I: Steady-state maintenance," J. Atmos.Sci, v.43:pp.585604,1986 .

[3] N.O.Renno, M.L.Barkett, and M.P.Larkin, "A simple thermodynamical theory for dust devils," J. Atmos.Sci, v.55, pp.3244-3252, 1998.

[4] N.O.Renno, H.B.Bluestein, "A simple theory for waterspouts," J. Atmos.Sci, v.58, pp.927-932, 2001.

[5] A.M.Nechayev, "A New Approach in Interpretation and Solving Certain Hydrodynamical Phenomena and Systems," Physical Science International Journal, v.11(1), pp. 1-11, 2016.

[6] A.Nechayev, "On the Bernoulli Equation in the Free Atmospere and Mechanism of Long-lived Vortex Formation," Physical Science International Journal, v.11(4), pp. 1-6, 2016

[7] L.Prandtl. Essentials of Fluid Dynamics, London, Blackie \& Son, 1952

[8] H.F.Hawkins, S.M.Imbembo, "The Structure of a Small, Intense Hurricane - Inez 1966.," Monthly Weather Review, v.104, pp.418442, 1976.

[9] G.Holland, "A revised Hurricane Pressure-Wind Model," Monthly Weather Review, v.136, pp. 3432-3436, 2007.

[10] J.Knaff, R.Zehr, "Reexamination of Tropical Cyclone Wind-Pressure Relationships," Weather and Forecasting, v.22, pp.71-88, 2007.

[11] C.Landsea, "Hurricane Structure: Theory and Diagnosis," presented on World meteorological Organization Workshop, 6 march, 2017

[12] V.H.Leverson, P.C.Sinclair, J.H.Golden, "Waterspout Wind, Temperature and Pressure Structure Deduced from Aircraft Measurements," Monthly Weather Review, v.105, pp.725-733, 1977.

[13] D.J.Novlan, W.M.Gray, "Hurricane-Spawned Tornadoes," Monthly Weather Review, v.102, pp.476-486, 1974. 


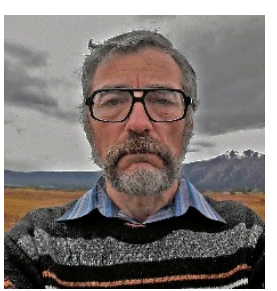

Andrei Nechayev. After graduating in 1977 MIREA (Russian Technological University), for 10 years he theoretically investigated the electrical instability in semiconductor devices and defended his thesis. According to the results of numerous expeditions to Kamchatka Peninsula he published 9 illustrated books, including "Miracles of Kamchatka Land" (5 editions in 1999-2015), "Kamchatka. Volcanoes Kingdom" (2008), "Kamchatka Fiery Heart" (2014). He is the author of a new theoretical description of the mechanism of eruptions of geysers and volcanoes. Since 2011 Dr. Nechayev is a researcher at Geographic Faculty of Lomonosov's Moscow State University.

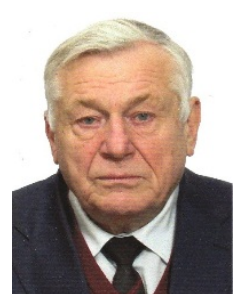

Hydromechanics."

Alexander Solovyev. Professor, Doctor of Phys. and Mathem. Sci., Member Acad. of Natur. Sci. (2003), Lomonosov's Moscow State University Associate Professor, Physical Faculty (1965- 1987). Professor Solovyev heads the Department of Renewable Sources Energy, Geographical Faculty, (from 1987 to the present). He is an author of more than 150 original articles and 10 books including the monograph: "A New Look at the Problem of Physical Acoustics, Seaweed Energy, and Physical 